\title{
Clinical Comparison of a Silicone Hydrogel and a Conventional Hydrogel Daily Disposable Contact Lens
}

\author{
Jason Miller' \\ Bradley Giedd ${ }^{2}$ \\ Lakshman N Subbaraman ${ }^{3}$ \\ 'EyeCare Professionals of Powell, Powell, \\ $\mathrm{OH}$, USA; ${ }^{2}$ Maitland Vision Center, \\ Maitland, FL, USA; ${ }^{3}$ Alcon Research, LLC, \\ Johns Creek, GA, USA
}

Purpose: To compare the subjective performances of verofilcon A daily disposable silicone hydrogel contact lenses (CLs) and etafilcon A hydrogel CLs.

Methods: Successful wearers of spherical soft CLs for distance correction were prospectively randomized to wear verofilcon A or etafilcon A lenses for 1 week and crossed over to the alternative lenses. The primary study objective was a comparison of distance visual acuity (VA). Exploratory endpoints included subjective overall lens preference (5-point scale) and subjective ratings (10-point scales) of end-of-day (EOD) vision, overall handling, insertion comfort, EOD comfort, overall quality of vision, overall comfort, vision throughout the day, lens handling at insertion, and lens handling at removal.

Results: Of 92 subjects (184 eyes), 46 each were randomized to verofilcon A or etafilcon A lenses and subsequently crossed over to the other lenses. Evaluation of distance VA showed that verofilcon A lenses were noninferior to etafilcon A lenses. Comparison of lens preference showed that $68(73.9 \%)$ subjects somewhat or strongly preferred verofilcon A lenses, whereas $21(22.9 \%)$ somewhat or strongly preferred etafilcon A lenses $(\mathrm{p}<0.0001)$. Mean \pm SD ratings of EOD vision $(8.6 \pm 1.5$ vs $7.7 \pm 1.9)$, overall handling $(8.7 \pm 1.5$ vs 6.9 $\pm 2.3)$, insertion comfort $(9.2 \pm 1.0$ vs $7.7 \pm 1.9)$, and EOD comfort $(8.0 \pm 1.9$ vs $7.0 \pm 2.2)$ were all significantly ( $\mathrm{p} \leq 0.0001$ each) higher for verofilcon A than for etafilcon A lenses. Mean \pm SD ratings of overall quality of vision $(8.9 \pm 1.2$ vs $8.2 \pm 1.8)$, overall comfort ( $8.6 \pm 1.5$ vs 7.4 $\pm 1.8)$, vision throughout the day $(8.9 \pm 1.3$ vs $8.1 \pm 1.8)$, lens handling at insertion $(9.0 \pm 1.4 \mathrm{vs}$ $6.9 \pm 2.5)$, and lens handling at removal ( $8.3 \pm 2.1$ vs $7.7 \pm 2.2)$ were also significantly higher for verofilcon A lenses. No subject experienced any ocular adverse events.

Conclusion: After 1 week of wear, the study population reported that ratings for subjective endpoints were significantly higher for verofilcon A lenses than for etafilcon A lenses.

Keywords: etafilcon A, subjective, verofilcon A, visual acuity

\section{Introduction}

Contact lens materials and design are constantly being improved to increase the accuracy of vision correction and to improve comfort and ease of handling, for both new and habitual wearers of contact lenses. ${ }^{1,2}$ Daily disposable contact lenses are intended to be worn during waking hours for a full day and to be discarded the same day after use. Wearing of daily disposable lenses eliminates the need for cleaning and/or disinfecting solutions, resulting in a higher compliance rate for lens replacement. ${ }^{3-6}$

Most contact lenses currently prescribed are soft contact lenses. In 2019, it was estimated that soft contact lenses accounted for about $87 \%$ of lens fits. ${ }^{7}$ Of the soft
Correspondence: Jason Miller EyeCare Professionals of Powell, 97II Sawmill Parkway, Powell, OH, 43065, USA Tel +I 6I4 793-0700

Fax +I 614 793-0084

Email millereyedoc@me.com;

drmiller@eyecarepowell.com 
contact lenses prescribed in 2019, about $40 \%$ were daily disposable contact lenses. Discontinuation of lens wear is a major problem, especially in new wearers of contact lenses. One study reported that $22 \%$ of new contact lens wearers permanently discontinued wear during the first year; with $50 \%$ of these discontinuing during the first 3 months of wear. ${ }^{2}$ The primary reason given for discontinuation of contact lens wear was poor vision quality, followed by poor comfort, especially at the end of the day, and difficulty handling of the lens.

Verofilcon A is a highly breathable silicone hydrogel contact lens material, with a $\mathrm{Dk} / \mathrm{t} 35^{\circ} \mathrm{C}$ of $100 \times 10^{-11}\left(\mathrm{~cm}^{2} / \mathrm{sec}\right)$ $(\mathrm{mL} \mathrm{O} / 2 / \mathrm{mL} \times \mathrm{mmHg}){ }^{8}$ The core of verofilcon A lenses has a $51 \%$ water content and a modulus of $0.6 \mathrm{MPa}$, allowing for easy handling; and the surface of these lenses has a permanent level of moisture with $>80 \%$ water content. Verofilcon A contact lenses were approved for wear by the US Food and Drug Administration in 2019. These lenses were found to confer excellent visual acuity, with subjects consistently providing high ratings for vision quality, comfort, and handling. ${ }^{9}$

Etafilcon A is a widely used, high-quality and wellaccepted conventional hydrogel contact lens material that have been marketed for a long period of time. ${ }^{10-12}$ These lenses are regarded as a benchmark for safety, efficacy, and comfort and are often used as a comparator in clinical trials with other lenses. Etafilcon A lenses contain the wetting agent polyvinylpyrrolidone, which mimics the natural tear film, and have a water content of $58 \%$, with an excellent safety profile. Evaluation of hypoxic stress showed that etafilcon A lenses were noninferior to two types of silicone hydrogel lenses, lotrafilcon B and comfilcon A. ${ }^{13}$ Etafilcon A daily disposable lenses were selected for comparison with verofilcon A lenses because of their design and replacement schedule (Table 1).

The present clinical study was designed to compare the objective and subjective performance of verofilcon A contact lenses with those of already marketed etafilcon
A daily disposable contact lenses. The primary objective of this study was to demonstrate that distance visual acuity (VA) is noninferior in subjects wearing verofilcon A contact lenses compared with the same subjects wearing etafilcon A contact lenses. The key subjective endpoints reported here by wearers of verofilcon A and etafilcon A contact lenses included overall preference, end-of-day vision (EOD), overall handling, insertion comfort and EOD comfort. Other subjective comparisons included overall quality of vision, overall comfort, vision throughout the day, lens handling at insertion, and lens handling at removal.

\section{Materials and Methods \\ Study Design}

This was a prospective, randomized, bilateral crossover, double-masked, controlled clinical study. Subjects were enrolled at five study centers in the US. Subjects were expected to attend three study visits over approximately 14-20 days (7-10 days for each lens type) from May 3 to June 24 of 2019. The study was confirmed with the ethical principles of the Declaration of Helsinki and the International Council for Harmonisation (ICH) E6 Good Clinical Practice (GCP) Consolidated Guidelines. The study protocol was approved by a central institutional review board (Sterling IRB, Atlanta, GA) and all subjects provided written informed consent. The ClinicalTrials.gov identifier number is NCT03888469.

\section{Inclusion Criteria}

Subjects aged $\geq 18$ years were included if they had eyes without active ocular disease and were successful wearers of spherical soft contact lenses for distance correction during the 3 months prior to enrolment, with lenses worn for a minimum 5 days/week. Other inclusion criteria included a best corrected VA (BCVA) of 20/25 or better

Table I Properties of Verofilcon A and Etafilcon A Lenses

\begin{tabular}{|l|l|l|}
\hline & Verofilcon A & Etafilcon A \\
\hline Refractive index (hydrated) & 1.4 & 1.4 \\
Light transmittance & $\geq 90 \% @ 640 \mathrm{~nm},-3.00 \mathrm{D}$ & $\geq 85 \% @ 640 \mathrm{~nm},-3.00 \mathrm{D}$ \\
Oxygen permeability (Dk) & $90 \times 10^{-11}\left(\mathrm{~cm}^{2} / \mathrm{sec}\right)(\mathrm{mL} \mathrm{O} / \mathrm{mL} \times \mathrm{mmHg})$ & $21.4 \times 10^{-11}\left(\mathrm{~cm}^{2} / \mathrm{sec}\right)(\mathrm{mL} \mathrm{O} / \mathrm{mL} \times \mathrm{mmHg})$ \\
Water content & $51 \%$ by weight in normal saline & $58 \%$ by weight in normal saline \\
Diameter & $14.2 \mathrm{~mm}$ & $14.2 \mathrm{~mm}$ \\
Spherical power range & -1.00 to $-6.00 \mathrm{D}$ (in $0.25 \mathrm{D}$ steps) & -1.00 to $-6.00 \mathrm{D}$ (in $0.25 \mathrm{D}$ steps) \\
Base curve & $8.3 \mathrm{~mm}$ & $8.5 \mathrm{~mm}$ \\
\hline
\end{tabular}


with a manifest cylinder $\leq 0.75 \mathrm{D}$ in each eye; a willingness and ability to return for the three study visits; the ability to wear contact lenses within a range of sphere power from -1.00 to $-6.00 \mathrm{D}$ and VA $20 / 25$ or better and provision of written informed consent.

\section{Exclusion Criteria}

Subjects were excluded if they were previous or current wearers of delefilcon A or etafilcon A lenses or if they were habitual wearers of extended-wear contact lenses during the 3 months prior to study enrolment. Subjects were also excluded if they had any anterior segment contraindication to lens wear; a history of ocular or intraocular surgery; pathologic dry eye that would, in the opinion of the investigator, preclude contact lens wear; or herpetic keratitis; or had ever used any systemic or ocular medications contraindicating contact lens wear.

\section{Study Protocol}

During the first visit, subjects were screened, randomized to verofilcon A or etafilcon A lenses, and dispensed these lenses. Subjects were instructed to wear these lenses for 10 hours per day (range, 8-16 hours) for $8(-1 \pm 2)$ days starting the following day. During the second visit, subjects were evaluated for all study objectives and were dispensed the alternative lenses. Subjects were instructed to wear these lenses for 10 hours per day (range, 8-16 hours) for $8(-1 \pm 2)$ days, starting the following day. During the third visit, subjects were evaluated for all study objectives.

\section{Statistical Analysis \\ Calculation of Sample Size}

Sample size calculation was based on a prior clinical study, which evaluated the performance of etafilcon $\mathrm{A}$ and three marketed soft contact lenses, including verofilcon A lenses [unpublished]. The primary objective of the present study was to demonstrate that distance VA is noninferior in subjects wearing verofilcon A contact lenses compared with the same subjects wearing etafilcon A contact lenses. To demonstrate noninferiority (margin $=0.05 \log$ MAR, $1 / 2$ line in Snellen) as a one-tailed hypothesis with $\alpha=0.05$, and using a standard deviation of $0.068,80 \%$ power could be attained with a sample size of 14 ( 7 per sequence). For overall preference, the sample size required to detect a difference between $77.3 \%$ and the hypothesized value of $50 \%$ was 32 (16 per sequence) at $80 \%$ power and a onesided $\alpha=0.01$, assuming all subjects indicate a preference.
For the other subjective ratings (EOD vision, overall handling, insertion comfort, EOD comfort), the sample sizes required to detect a difference of 1.0 at $80 \%$ power and a one-sided $\alpha=0.01$ ranged from 25 to 41 per sequence. Assuming a loss-to-follow up of $10 \%$, a minimum of 92 subjects was regarded as necessary.

\section{Analysis of Primary Effectiveness}

The primary endpoint, distance VA in logMAR units, was measured in each eye at each study visit and was analyzed with a mixed-effects repeated-measures model, using terms for lens, period and sequence, and accounting for within-subject correlations due to eye and the crossover design. The difference between verofilcon A and etafilcon A lenses and corresponding one-sided 95\% upper confidence limits (UCL) were compared, with noninferiority in distance VA defined as an upper confidence limit $<0.05$.

\section{Analysis of Exploratory Effectiveness}

Lens preference was determined after subjects wore the second set of lenses for 1 week. Subjects indicated whether they strongly preferred Lens 1; somewhat preferred Lens 1; had no preference; somewhat preferred Lens 2; or strongly preferred Lens 2. Overall preference was evaluated using the exact binomial test. Proportions were compared and the corresponding one-sided p-value was calculated. Preferences for either lens type were determined for subjects who reported a preference and compared with the hypothesized preference of $50 \%$.

Subjective rating questionnaires were completed at the end of each 1-week lens wear period (visits 2 and 3) for EOD vision, overall handling, insertion comfort and EOD comfort, as well as for overall quality of vision, overall comfort, vision throughout the day, lens handling at insertion and lens handling at removal. Insertion comfort, defined as comfort during the first 2 minutes after lens insertion, was collected in diaries by each subject on day 1 in the morning and at the 1-week follow-up, also in the morning. Each of the above endpoints was rated on a 10 point scale, ranging from 1 (poor) to 10 (excellent), with only whole numbers allowed. Subjects provided one rating for both eyes. Ratings were compared by the Holm method, a mixed-effects repeated-measures model, using terms for lens, period and sequence, and accounting for within-subject correlations due to the crossover design. Also evaluated were the percentages of subjects with ratings of $\geq 9$ for each lens material on each of these exploratory parameters. One-sided p-values were calculated, with 
significantly higher ratings for verofilcon A than etafilcon A lenses on each subjective parameter defined as a onesided p-value $<0.01$ in favor of verofilcon A lenses.

\section{Results}

A total of 92 subjects (184 eyes) were enrolled in this study at five study centers in the United States. Forty-six subjects were randomized to wear verofilcon A followed by etafilcon A lenses, and 46 to wear etafilcon A followed by verofilcon A lenses (Figure 1). The mean (SD) age overall for subjects was 32.6 (8.2) years (range, 18-50 years) and the majority of subjects were female $(64.1 \%)$, white $(90.2 \%)$, and not of Hispanic or Latino ethnicity (89.1\%). All subjects completed the study. The demographic characteristics were similar in the two groups of subjects (Table 2).

\section{Primary Objective: Noninferiority of Verofilcon $A$ to Etafilcon A Lenses}

The least squares means (LSM) (standard error [SE]) distance VA was $-0.13(0.008) \operatorname{logMAR}$ units for verofilcon A lenses and $-0.12(0.008) \log$ MAR units for etafilcon A lenses, with an LSM (SE) difference in VA of -0.00 (0.004) $\operatorname{logMAR}$ units and a UCL for the difference of 0.00 . Because the $95 \%$ UCL was less than the pre-

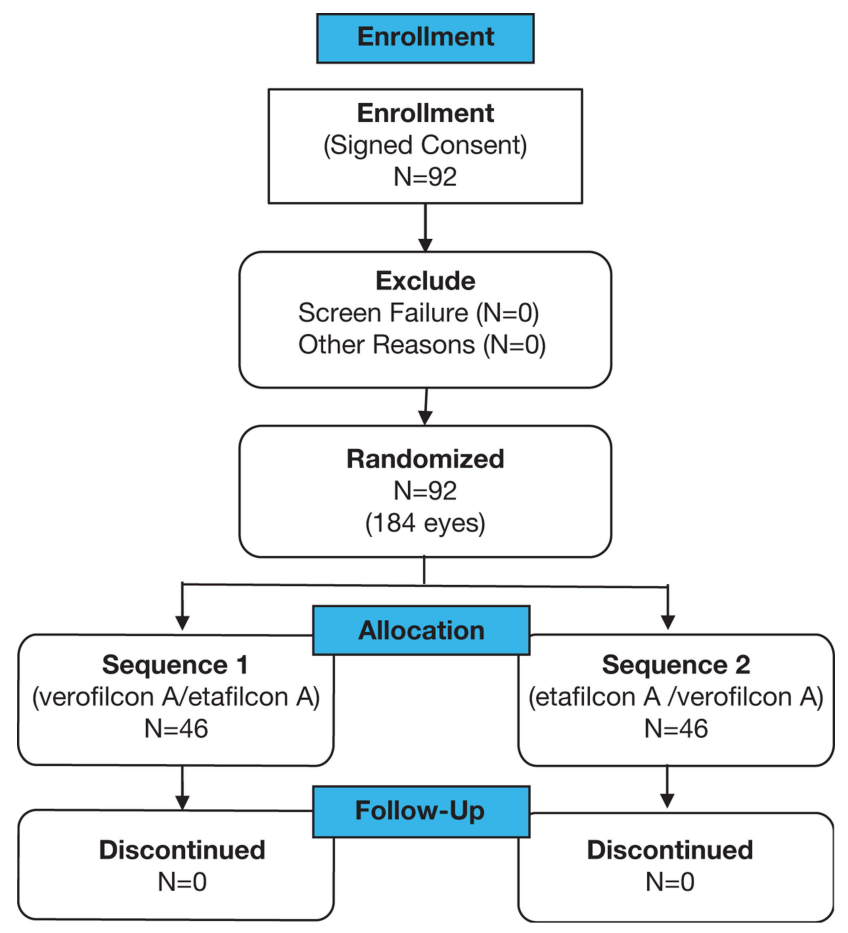

Figure I Study population diagram.
Table 2 Demographic Characteristics of Study Subjects by Sequence of Lens Wear

\begin{tabular}{|l|l|l|l|}
\hline & $\begin{array}{l}\text { Verofilcon A/ } \\
\text { Etafilcon A } \\
(\mathbf{n}=\mathbf{4 6})\end{array}$ & $\begin{array}{l}\text { Etafilcon A/ } \\
\text { Verofilcon A } \\
\mathbf{( n = 4 6 )}\end{array}$ & $\begin{array}{l}\text { Overall } \\
\mathbf{( N = 9 2 )}\end{array}$ \\
\hline Age, years, mean \pm SD & $33.6 \pm 8.5$ & $31.5 \pm 7.8$ & $32.6 \pm 8.2$ \\
\hline $\begin{array}{l}\text { Sex, } \mathrm{n}(\%) \\
\text { Male } \\
\text { Female }\end{array}$ & $15(32.6)$ & $18(39.1)$ & $33(35.9)$ \\
\hline $\begin{array}{l}\text { Race, } \mathrm{n}(\%) \\
\text { White }\end{array}$ & $31(67.4)$ & $28(60.9)$ & $59(64.1)$ \\
Black & $43(93.5)$ & $40(87.0)$ & $83(90.2)$ \\
Asian & $2(4.3)$ & $4(8.7)$ & $6(6.5)$ \\
Other & $0(0.0)$ & $1(2.2)$ & $1(1.1)$ \\
\hline Ethnicity, n (\%) & $1(2.2)$ & $1(2.2)$ & $2(2.2)$ \\
Hispanic or Latino & $2(4.3)$ & $8(17.4)$ & $10(10.9)$ \\
Not Hispanic or Latino & $44(95.7)$ & $38(82.6)$ & $82(89.1)$ \\
\hline
\end{tabular}

specified margin of 0.05 , verofilcon A lenses were noninferior to etafilcon A lenses.

\section{Secondary Endpoints}

Of the 92 subjects, 68 (73.9\%) preferred or strongly preferred verofilcon A lenses, with nearly five times as many strongly preferring verofilcon A to etafilcon A; 21 (22.8\%) preferred or strongly preferred etafilcon A lenses, and 3 (3.3\%) expressed no preference (Figure 2). Of the 89 subjects who reported a preference, $68(76.4 \%)$ preferred verofilcon A than the etafilcon A lenses, significantly higher than the hypothesized 50.0\% $(\mathrm{p}<0.0001$ based on an exact binomial test).

\section{Subjective Evaluations}

Higher percentages of subjects provided verofilcon A than etafilcon A lenses with ratings $\geq 9$ for EOD vision (62.0\% vs $39.1 \%$ ), overall handling ( $67.4 \%$ vs $25.0 \%$ ), insertion comfort $(84.8 \%$ vs $37 \%)$ and EOD comfort $(53.3 \%$ vs $27.2 \%$ ). In addition, higher percentages of subjects provided verofilcon $A$ than etafilcon A lenses with ratings $\geq 9$ for overall quality of vision $(77.2 \%$ vs $52.2 \%)$, vision throughout the day $(73.9 \%$ vs $47.8 \%)$, lens handling at insertion $(76.1 \%$ vs $33.7 \%)$, lens handling at removal (60.9\% vs $43.5 \%)$ and overall comfort (66.3\% vs $32.6 \%)$.

Mean \pm SD ratings of lens handling at insertion $(9.0 \pm$ 1.4 vs $6.9 \pm 2.5, \mathrm{p}<0.0001)$ and overall comfort $(8.6 \pm 1.5$ vs $7.4 \pm 1.8, \mathrm{p}<0.0001)$ were statistically significantly higher for verofilcon A than for etafilcon A lenses 


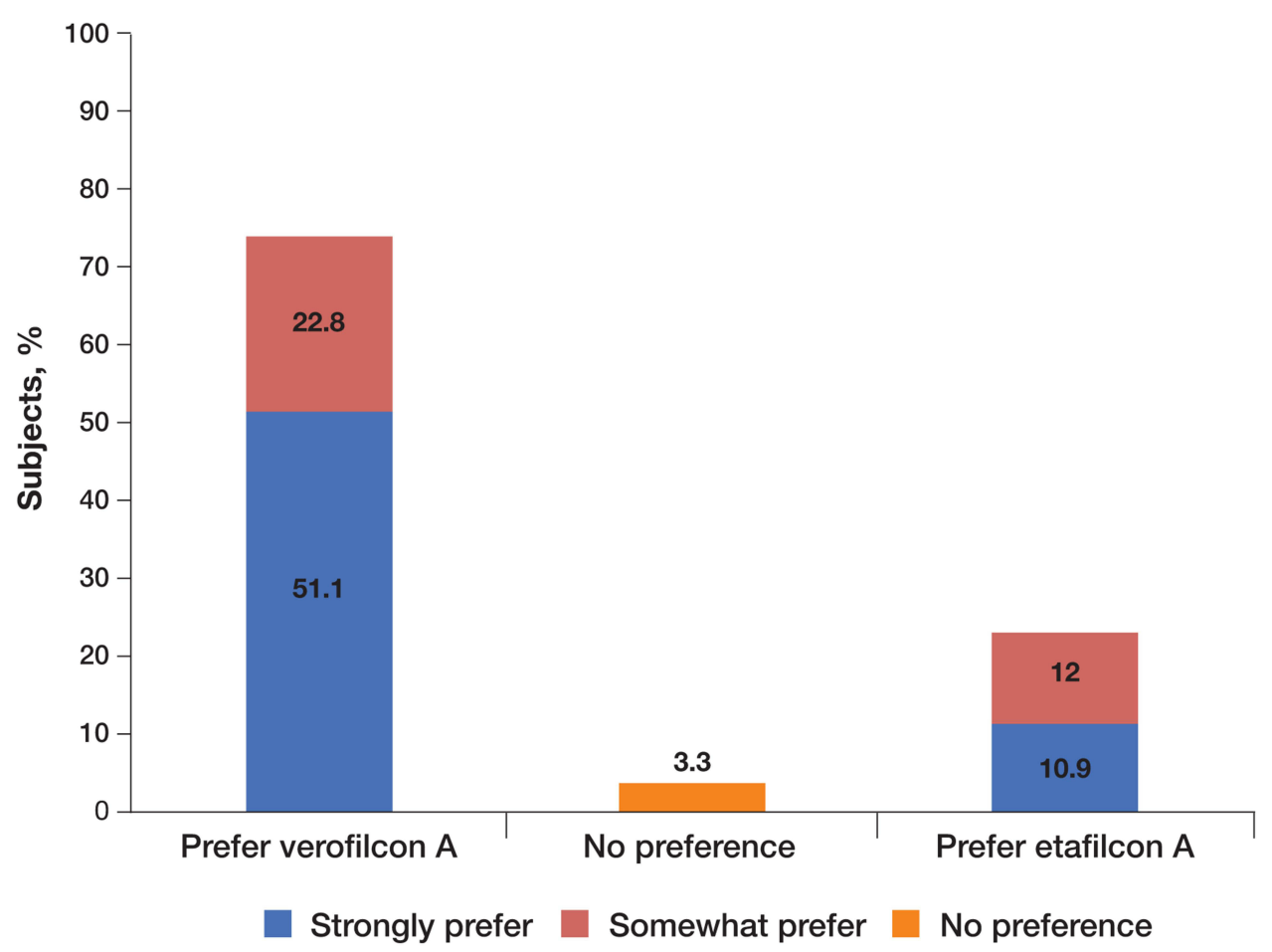

Figure 2 Overall preference of study subjects for verofilcon A and etafilcon A lenses.

(Table 3). Mean \pm SD ratings of overall quality of vision $(8.9 \pm 1.2$ vs $8.2 \pm 1.8, \mathrm{p}=0.0002)$, vision throughout the day $(8.9 \pm 1.3$ vs $8.1 \pm 1.8, \mathrm{p}<0.0001)$, and lens handling at removal $(8.3 \pm 2.1$ vs $7.7 \pm 2.2, \mathrm{p}=0.0149)$ were also higher for verofilcon A than for etafilcon A lenses. In addition, the mean \pm SD ratings of EOD vision $(8.6 \pm 1.5$ vs $7.7 \pm 1.9, \mathrm{p}=0.0001)$, overall handling $(8.7 \pm 1.5$ vs $6.9 \pm 2.3, p<0.0001)$, insertion comfort

Table 3 Subjective Ratings of Verofilcon A and Etafilcon A Lenses for Overall Quality of Vision, Overall Comfort, Vision Throughout the Day, Lens Handling at Insertion and Lens Handling at Removal, End-of-Day Vision, Overall Handling, Insertion Comfort, and End-of-Day Comfort

\begin{tabular}{|c|c|c|c|}
\hline & \multicolumn{2}{|c|}{ Mean \pm SD Ratings } & \multirow[t]{2}{*}{ P-value } \\
\hline & Verofilcon $\mathbf{A}$ & Etafilcon A & \\
\hline Overall quality of vision & $8.9 \pm 1.2$ & $8.2 \pm 1.8$ & 0.0002 \\
\hline Overall comfort & $8.6 \pm 1.5$ & $7.4 \pm 1.8$ & $<0.0001$ \\
\hline Vision throughout the day & $8.9 \pm 1.3$ & $8.1 \pm 1.8$ & $<0.0001$ \\
\hline Lens handling at insertion & $9.0 \pm 1.4$ & $6.9 \pm 2.5$ & $<0.0001$ \\
\hline Lens handling at removal & $8.3 \pm 2.1$ & $7.7 \pm 2.2$ & 0.0149 \\
\hline End-of-day vision & $8.6 \pm 1.5$ & $7.7 \pm 1.9$ & 0.0001 \\
\hline Overall handling & $8.7 \pm 1.5$ & $6.9 \pm 2.3$ & $<0.0001$ \\
\hline Insertion comfort & $9.2 \pm 1.0$ & $7.7 \pm 1.9$ & $<0.0001$ \\
\hline End-of-day comfort & $8.0 \pm 1.9$ & $7.0 \pm 2.2$ & 0.0001 \\
\hline
\end{tabular}

(9.2 \pm 1.0 vs $7.7 \pm 1.9, \mathrm{p}<0.0001)$, and EOD comfort (8.0 \pm 1.9 vs $7.0 \pm 2.2, p=0.0001$ ) were all significantly higher for verofilcon A than for etafilcon A lenses.

\section{Safety Outcomes}

None of the subjects experienced any serious adverse events or ocular adverse events during the study period, and none experienced any clinically significant biomicroscopy findings.

\section{Discussion}

The present study compared the objective and subjective performances of verofilcon A contact lenses with those of etafilcon A daily disposable contact lenses. The primary objective of this study, that distance VA was noninferior in subjects wearing verofilcon A contact lenses than in the same subjects wearing etafilcon A contact lenses, was met, based on a noninferiority margin of $0.05 \log$ MAR units.

Analysis of key exploratory variables showed that the percentages of subjects rating lenses as $\geq 9$ (of 10) for EOD vision, overall handling, insertion comfort, and EOD comfort were higher for verofilcon A than etafilcon A lenses. LSM (SE) ratings for EOD vision, overall handling, insertion comfort, and EOD comfort were significantly higher for verofilcon A than etafilcon A lenses. Using the Holm 
method to adjust for multiplicity showed that the verofilcon A lenses were rated significantly higher than etafilcon A lenses with respect to overall preference, EOD vision, overall handling, insertion comfort, and EOD comfort.

Analysis of other exploratory effective endpoints showed that the percentages of subjects rating lenses as $\geq 9$ (of 10) for overall quality of vision, overall comfort, vision throughout the day, lens handling at insertion on day 7 and lens handling at removal were also higher for verofilcon A than etafilcon A lenses.

To understand patient and eye care professional satisfaction in real-world conditions, a survey was conducted with verofilcon A daily disposable contact lenses; 218 current wearers of daily disposable contact lenses and 129 new wearers of contact lenses wore verofilcon A lenses for at least 8 hours/day for 1 week. ${ }^{14}$ Subjects completed questionnaires before and after wearing these lenses for 1 week. New contact lens wearers reported improvements in their day-today quality of life. Of the 172 current contact lens wearers who expressed a preference for either verofilcon A lenses or their previous contact lenses, $81 \%$ expressed a preference or strong preference for verofilcon A lenses, a preference influenced by long-lasting comfort, less feeling of dryness, allday comfort and longer wear time.

As part of this same evaluation, 31 eye care professionals who fitted up to 10 current and 10 new wearers with verofilcon A lenses were surveyed before and after the study. ${ }^{14}$ Of these professionals, 17 (54\%) switched from their most preferred brand of contact lenses to verofilcon A lenses, with most stating that switching made them feel confident that they recommended the best brand of contact lenses to their patients.

This study had a few limitations, including its shortterm nature. Another limitation was the inability to completely mask the staff to lens assignment as an experienced ECP will be able to determine the lens type based on the tint of the contact lenses. Although all subjects and personnel conducting the study were masked to the study lens assignment, the lenses were dispensed by a qualified unmasked study staff member such that the subjects and investigators remained masked.

In conclusion, this study showed that verofilcon A contact lenses were noninferior to etafilcon A lenses as determined by distance VA. After 1 week of wear, the study population gave significantly higher ratings to verofilcon A than etafilcon A lenses, as determined by overall preference and other subjective endpoints, including EOD vision, overall handling, insertion comfort, EOD comfort, overall quality of vision, overall comfort, vision throughout the day, lens handling at insertion and lens handling at removal. Further clinical trials are warranted to evaluate long-term use of verofilcon A contact lenses.

\section{Data Sharing Statement}

Due to varying rights of individuals and contractual rights of parties involved, Alcon does not make a practice of sharing datasets.

\section{Acknowledgments}

The authors wish to thank their co-investigators: Bradley Hines, OD (Optometry Group, Memphis, TN), Mark Perry, OD (Vision Health Institute, Orlando, Fl) and Jon Walker, OD, MS (Dr Ted Brink and Associates, Jacksonville, FL).

\section{Funding}

Dr Lakshman N. Subbaraman is an employee of Alcon Research, LLC. Editorial support was provided by BelMed, Inc. with funding by Alcon Research, LLC.

\section{Disclosure}

Dr Lakshman N. Subbaraman is an employee of Alcon Research, LLC. The authors report no other conflicts of interest in this work.

\section{References}

1. Nichols JJ, Willcox MD, Bron AJ, et al.; members of the TFOS International Workshop on Contact Lens Discomfort. The TFOS International workshop on contact lens discomfort: executive summary. Invest Ophthalmol Vis Sci. 2013;54(11):TFOS7-TFOS13. doi:10.1167/iovs.13-13212.

2. Sulley A, Young G, Hunt C, McCready S, Targett MT, Craven R. Retention rates in new contact lens wearers. Eye Cont Lens. 2018;44 (Suppl 1):S273-S282. doi:10.1097/ICL.0000000000000402

3. Dumbleton K, Woods C, Jones L, Fonn D, Sarwer DB. Patient and practitioner compliance with silicone hydrogel and daily disposable lens replacement in the United States. Eye Cont Lens. 2009;35 (4):164-171. doi:10.1097/ICL.0b013e3181ac4a8d

4. Dumbleton K, Richter D, Woods C, Jones L, Fonn D. Compliance with contact lens replacement in Canada and the United States. Optom Vis Sci. 2010;87(2):131-139. doi:10.1097/OPX.0b013e3181ca32dc

5. Morgan PB, Efron N, Toshida H, Nichols JJ. An international analysis of contact lens compliance. Cont Lens Anterior Eye. 2011;34 (5):223-228. doi:10.1016/j.clae.2011.08.001

6. Sulley A, Dumbleton K. Silicone hydrogel daily disposable benefits: the evidence. Cont Lens Anterior Eye. 2020;43(3):298-307. doi:10.1016/j.clae.2020.02.001

7. Morgan P, Woods CA, Tranoudis IG, et al. International contact lens prescribing in 2019. Cont Lens Spectrum. 2020;35(1):26-32.

8. Matthew J. Precision $1{ }^{\circledR}$ contact lenses with Smartsurface ${ }^{\circledR}$ technology: material properties, surface wettability and clinical performance; 2019. Available from: https://us.alconscience.com/wp-content/uploads/2019/ 08/1906A195-US-DP1-19-E-1306_DP1-White-Paper_singe_lr.pdf. Accessed January 6, 2020. 
9. Cummings S, Giedd B, Pearson C. Clinical performance of a novel daily disposable spherical contact lens. J Cont Lens Res Sci. 2020;4 (1):e23-e30. doi:10.22374/jclrs.v4i1.39

10. Chalmers RL, Hickson-Curran SB, Keay L, Gleason WJ, Albright R. Rates of adverse events with hydrogel and silicone hydrogel daily disposable lenses in a large post-market surveillance registry: the TEMPO Registry. Invest Ophthalmol Vis Sci. 2015;56(1):654-663. doi:10.1167/iovs.14-15582

11. Grobe GL 3rd, Valint PL Jr, Ammon DM Jr. Surface chemical structure for soft contact lenses as a function of polymer processing. J Biomed Mater Res. 1996;32(1):45-54. doi:10.1002/ (SICI)1097-4636(199609)32:1<45::AID-JBM6>3.0.CO;2-P
12. Efron N, Brennan NA, Chalmers RL, et al. Thirty years of 'quiet eye' with etafilcon A contact lenses. Cont Lens Anterior Eye. 2020;43 (3):285-297. doi:10.1016/j.clae.2020.03.015

13. Szczotka-Flynn LB, Debanne S, Benetz BA, Wilson T, Brennan N. Daily wear contact lenses manufactured in etafilcon A are noninferior to two silicone hydrogel lens types with respect to hypoxic stress. Eye Cont Lens. 2018;44(3):190-199. doi:10.1097/ICL.000000000 0000335

14. Grant T, Tang A. A survey of contact lens wearers and eye care professionals on satisfaction with a new smart-surface silicone hydrogel daily disposable contact lens. Clin Optom. 2020;12:9-15. doi:10.2147/OPTO.S233328
Clinical Ophthalmology

\section{Publish your work in this journal}

Clinical Ophthalmology is an international, peer-reviewed journal covering all subspecialties within ophthalmology. Key topics include: Optometry; Visual science; Pharmacology and drug therapy in eye diseases; Basic Sciences; Primary and Secondary eye care; Patient Safety and Quality of Care Improvements. This journal is indexed on PubMed

Submit your manuscript here: https://www.dovepress.com/clinical-ophthalmology-journal
Dovepress

Central and CAS, and is the official journal of The Society of Clinical Ophthalmology (SCO). The manuscript management system is completely online and includes a very quick and fair peer-review system, which is all easy to use. Visit http://www.dovepress.com/ testimonials.php to read real quotes from published authors. 